\title{
Numerical simulations of the first harmonic kink mode of vertical oscillations of a solar coronal loop
}

\author{
A. Wasiljew and K. Murawski
}

\author{
Group of Astrophysics and Gravity Theory, Institute of Physics, UMCS, ul. Radziszewskiego 10, 20-031 Lublin, Poland \\ e-mail: awasiljew@gmail.com
}

Received 23 September 2008 / Accepted 16 January 2009

\section{ABSTRACT}

\begin{abstract}
Aims. We investigate impulsive excitation mechanisms of the first harmonic fast magnetoacoustic kink mode of vertical oscillations in a solar coronal loop, which is approximated by either a straight slab or a curved arcade slab. Methods. We solve numerically the full set of nonlinear ideal two-dimensional magnetohydrodynamic equations and analyze temporal and spatial wave signatures to infer characteristic features, such as waveperiods and node positions.

Results. We show that an initial velocity pulse located off-center excites the first harmonic and fundamental modes, which result in a movement of the node around the loop apex. These results are verified by the analytical findings for an elastic string.

Conclusions. Since the fundamental mode acquires more energy than its first harmonic, this explains why the latter is so rarely detected within a coronal loop.
\end{abstract}

Key words. magnetohydrodynamics (MHD) - Sun: corona - Sun: oscillations

\section{Introduction}

The development of highly sensitive instruments such as SUMER (SOHO), TRACE, and Hinode resulted in the detection of various oscillations in solar coronal loops. Two types of waves were observed: (a) propagating (De Moortel et al. 2002) and standing (Wang et al. 2005) slow magnetoacoustic waves, and (b) fast magnetoacoustic standing waves: horizontal kink (Nakariakov et al. 1999; Aschwanden et al. 1999; Schrijver et al. 1999), vertical kink (Wang \& Solanki 2004; Ofman \& Wang 2008), and sausage (Nakariakov et al. 2003; Aschwanden et al. 2004) waves. Because of the insufficient temporal resolution of the observational data, it is often difficult to identify which mode of the oscillations is excited (Aschwanden et al. 2004). Therefore, despite of a large number of observations, most data is interpreted in terms of a fundamental mode and, only several events of harmonic modes have been reported. In particular, two events of the first harmonic $(n=2)$ of the horizontal kink oscillations were detected by Verwichte et al. (2004) in a post-flare arcade loop. However, in both of these events this mode coexisted with the fundamental mode, so no pure higher harmonic mode was observed. De Moortel \& Brady (2007) observed higher harmonic horizontal kink modes $(n=2$ and $n=4(5)$ ). Van Doorsselaere et al. (2007) found in TRACE data two waveperiods that were consistent with the waveperiods of the fundamental and first harmonic mode of fast magnetoacoustic kink oscillations.

Oscillations of magnetic loops can be studied both analytically and numerically. In this context, Díaz et al. (2006) studied analytically the fundamental and first harmonic modes of the vertical kink oscillations. They found that the first harmonic mode corresponds in a straight cylinder to the mode $n=2$ (Roberts et al. 1984). As a result of higher temporal and spatial resolutions in numerical experiments than in observations, a detection of higher harmonic oscillations should be more likely in a numerical data set. Brady \& Arber (2005) performed numerical experiments in which higher harmonics of vertical kink oscillations were excited by a footpoint velocity driver, while Ofman (2007) and Gruszecki et al. (2008), among others, triggered impulsively the fundamental mode.

The current work is motivated by the launch of the missions STEREO and Hinode, which provide observational data of far higher spatial and temporal resolution (Ofman \& Wang 2008). As a result, detection of higher harmonic modes in these data becomes likely. Since few reports of the observation of higher harmonic modes exists, our goal is to explain why these modes are so rarely observed within coronal loops.

This paper is organized as follows. The analytical findings for elastic string oscillations are shown in Sect. 2. The mathematical and numerical models are described in Sect. 3. The numerical results are presented and discussed in Sect. 4. This paper is concluded by a presentation of the main results in Sect. 5 .

\section{Fundamental and first harmonic modes of string oscillations}

We consider now the fundamental and first harmonic modes of elastic string oscillations which are present simultaneously in a system. This case will serve for comparison purposes with the oscillations in more realistic systems such as straight and curved slabs discussed in the following sections. These modes are represented by wave functions $z_{1}(x, t)$ and $z_{2}(x, t)$, while their interference state is denoted by $z(x, t)$, i.e.,

$$
\begin{aligned}
z(x, t) & =z_{1}(x, t)+z_{2}(x, t), \\
z_{n}(x, t) & =a_{n} \sin \left(k_{n} x\right) \sin \left(\omega_{n} t\right), \quad n=1,2 .
\end{aligned}
$$

Here subscripts 1 and 2 correspond respectively to the fundamental $(n=1)$ and first harmonic $(n=2)$ modes. Wave 


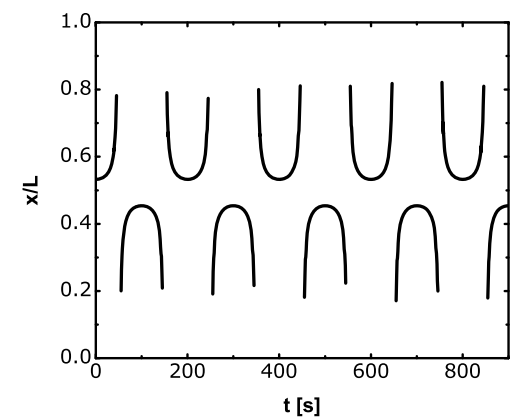

Fig. 1. Position of the node for two modes of oscillations, given by Eq. (6).

wavenumbers $k_{1}$, and $k_{2}$ and angular frequencies $\omega_{1}$, and $\omega_{2}$ are given as

$k_{1}=\pi / L, \quad \omega_{1}=V_{\mathrm{A}} k_{1}$,

$k_{2}=2 k_{1}, \quad \omega_{2}=2 \omega_{1}$,

where $V_{\mathrm{A}}$ is the wave speed and $L$ is the string length. We choose $V_{\mathrm{A}}=1 \mathrm{Mm} \mathrm{s}^{-1}$ and $L=100 \mathrm{Mm}$ for a numerical representation and assume that the string ends $(x=0$ and $x=L)$ are fixed in time, such that $z_{n}=0, n=1,2$, there.

It is noteworthy that a node of these oscillations is not fixed and instead it moves around the point $x=L / 2$. We derive the position of this node by requiring that

$z(x, t)=a \sin \left(k_{1} x\right) \sin \left(\omega_{1} t\right)\left[1+8 \cos \left(k_{1} x\right) \cos \left(\omega_{1} t\right)\right]=0$.

For simplicity reasons, we have assumed that the first harmonic $(n=1)$ oscillations occur with larger amplitude, i.e., $a_{1}=2 a_{2}=$ $2 a$. Hence, we find that the position of the node, $x(t)$, varies in time according to

$$
\begin{aligned}
x(t)= & \frac{L}{\pi} \arccos \left[-\frac{1}{8 \cos \left(\pi V_{\mathrm{A}} t / L\right)}\right], \\
& t \neq(j+1 / 2) \frac{L}{V_{\mathrm{A}}}, \quad j=0,1,2, \ldots .
\end{aligned}
$$

Figure 1 illustrates this formula. Initially at $t=0$, the node is settled at $x \simeq 0.5 L$. Its position moves in time until at $t=L /\left(2 V_{\mathrm{A}}\right)$ the node has moved asymptotically to the point $x=L$. The node reappears later on at $x=0$ and is then shifted to the central point of the string, $x=L / 2$. The entire process repeats cyclically in time and as a result the node oscillates about the central point, $x=L / 2$.

Time signatures for interference of the two oscillations of Eq. (1) are displayed in the top panels of Fig. 2. These time signatures correspond to wave signals which are collected in time at $x=L / 4$ (left panel) and at $x=L / 2$ (right panel). From the definition of $z_{1}(x, t)$, it is clear that the mode $n=1$ is best traced in a wave signal that is collected at $x=L / 2$, where $z_{1}(L / 2, t)$ attains extrema and $z_{2}(L / 2, t)=0$. We set a detection point at the location of the antinode of the pure first harmonic mode, $x=L / 4$. The bottom panels of Fig. 2, exhibits the corresponding Fourier power spectra for these time signatures. It is clear that the signal collected at $x=L / 4$ results from two waveperiods $P_{1}=200 \mathrm{~s}$ and $P_{2}=100 \mathrm{~s}$ (Fig. 2, bottom left panel), while the time signature collected at $x=L / 2$ is associated with only $P_{1}$ (bottom right panel). Here, the waveperiods follow the analytical formula

$P_{n}=\frac{2 L}{n V_{\mathrm{A}}}$,

where $n$ is the mode number. The fundamental (first harmonic) mode corresponds to $n=1(n=2)$.
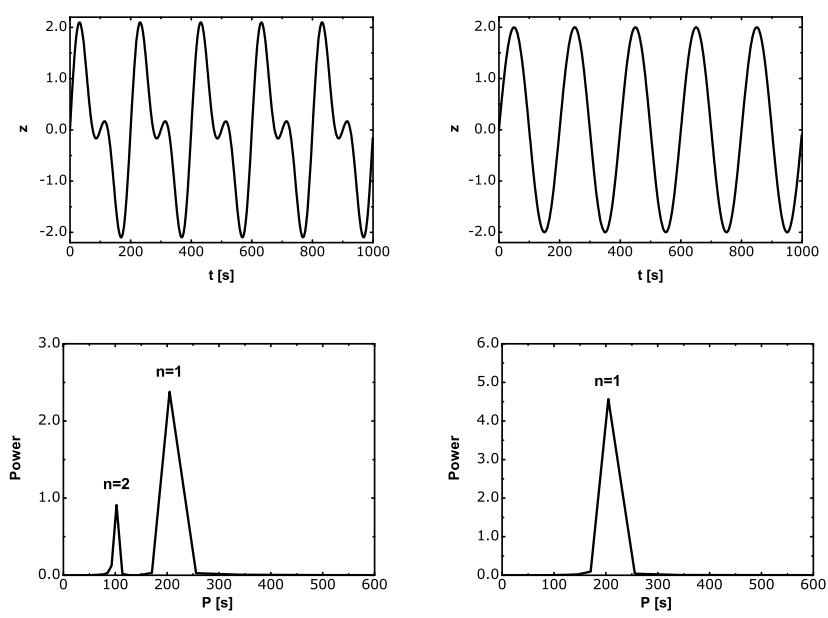

Fig. 2. Time signatures of $z(L / 4, t)$ (top left panel) and $z(L / 2, t)$ (top right panel) of Eq. (1) and the corresponding Fourier power spectra (bottom panels).

\section{Mathematical and numerical models of slabs}

We consider a magnetized plasma with polytropic index $\gamma=5 / 3$ and neglect gravitational stratification and non-ideal effects. As a result, we model coronal plasma with the ideal magnetohydrodynamic equations:

$\frac{\partial \varrho}{\partial t}+\nabla \cdot(\varrho \boldsymbol{V})=0$

$\varrho \frac{\partial \boldsymbol{V}}{\partial t}+(\varrho \boldsymbol{V} \cdot \nabla) \boldsymbol{V}=-\nabla p+\frac{1}{\mu}(\nabla \times \boldsymbol{B}) \times \boldsymbol{B}$,

$\frac{\partial E}{\partial t}+\nabla \cdot\left[\left(E+p+\frac{B^{2}}{2 \mu}\right) \boldsymbol{V}-\frac{\boldsymbol{B}(\boldsymbol{B} \cdot \boldsymbol{V})}{\mu}\right]=0$

$\frac{\partial \boldsymbol{B}}{\partial t}=\nabla \times(\boldsymbol{V} \times \boldsymbol{B})$,

$\nabla \cdot \boldsymbol{B}=0$.

Here $\mu$ is the magnetic permeability, $\varrho$ is mass density, $\boldsymbol{V}$ is flow velocity, $p$ is gas pressure, $\boldsymbol{B}$ is magnetic field, and the plasma energy density is expressed as

$E=\frac{\varrho V^{2}}{2}+\frac{p}{\gamma-1}+\frac{B^{2}}{2 \mu}$.

We consider both a curved slab and a straight slab and limit our discussion to a 2D magnetically structured solar corona with plasma quantities independent of the spatial coordinate $y$, $\partial / \partial y=0$, and neglect the polarization along the $y$-direction by setting $V_{y}=B_{y}=0$. A coronal slab is settled in a motionless environment $(\boldsymbol{V}=0)$. From the momentum Eq. (10), it follows that, for a constant equilibrium pressure ( $p_{\mathrm{e}}=$ const.), the Lorentz force must vanish. For a straight slab, this condition is satisfied by the equilibrium magnetic field components

$\boldsymbol{B}_{\mathrm{e}}=B_{0}[1,0,0]$,

where $B_{0}$ is the magnetic field at the reference level $z=0$. For a curved slab, we define an arcade magnetic field to be

$\boldsymbol{B}_{\mathrm{e}}=B_{0}\left[\cos \left(x / \Lambda_{\mathrm{B}}\right), 0,-\sin \left(x / \Lambda_{\mathrm{B}}\right)\right] \mathrm{e}^{-z / \Lambda_{\mathrm{B}}}$,

where $\Lambda_{\mathrm{B}}=2 L / \pi$ is the magnetic scale height, and $L=100 \mathrm{Mm}$ is the horizontal half-width of the arcade. The background mass 
density of the corona $\varrho_{\mathrm{e}}$ can be chosen arbitrary: for the straight (curved) slab, we assume that it decays with height $\left(\varrho_{\mathrm{e}}=\varrho_{\mathrm{e} 0}=\right.$ const.),

$\varrho_{\mathrm{e}}(z)=\varrho_{\mathrm{e} 0} \exp (-z / \Lambda)$.

Here $\varrho_{\mathrm{e} 0}=10^{-12} \mathrm{~kg} \mathrm{~m}^{-3}$ is a mass density at the reference level $z=0$ and $\Lambda$ is chosen to equal to $\Lambda_{B} / 2$. As a result the background Alfvén speed is constant, $V_{\mathrm{A}}(z)=\left|\boldsymbol{B}_{\mathrm{e}}\right| / \sqrt{\mu \varrho_{\mathrm{e}}}=$ $1 \mathrm{Mm} \mathrm{s}^{-1}$ for the straight slab but decays with height for the curved slab.

We also define $c_{s \mathrm{e}}=0.1 V_{\mathrm{A}}(z=0)$, where $c_{s \mathrm{e}}=\sqrt{\gamma p_{\mathrm{e}} / \varrho_{\mathrm{e} 0}}$ is the sound speed at $z=0$. A slab is embedded in the equilibrium structures in such a way that its edges follow two specific magnetic field lines. The straight slab length is $L=100 \mathrm{Mm}$ and its width is $4 \mathrm{Mm}$. The curved slab does not have a perfect circular shape, but its average radius and length can be estimated as $70 \mathrm{Mm}$ and $190 \mathrm{Mm}$, respectively. The mass density is enhanced in the slab compared to the ambient medium. We define the mass density contrast to be $d=\varrho_{\mathrm{i}} / \varrho_{\mathrm{e}}=10$, where $\varrho_{\mathrm{i}}$ denotes the mass density within the slab and $\varrho_{\mathrm{e}}$ corresponds to the ambient medium. Other curved slab parameters (footpoint separation, $1.4 \mathrm{~L}$, and a half-width of the slab at its footpoint, $0.025 \mathrm{~L}$ ) are chosen to ensure consistency with the model of Gruszecki et al. (2008).

We implement dense photosphere-like layers; for the straight slab, these layers are located at the left- and right-sides of the simulation region and for the curved slab a single layer is located at the bottom of a simulation region. For the straight slab, we have

$$
\begin{aligned}
\varrho_{0}(x)= & \varrho_{\mathrm{e} 0}+\frac{1}{2} \varrho_{\mathrm{e} 0}\left(d_{\mathrm{ph}}-1\right)\left\{\left[1-\tanh \left(\frac{x}{s_{\mathrm{ph}}}\right)\right]\right. \\
& \left.+\left[1-\tanh \left(-\frac{x-L}{s_{\mathrm{ph}}}\right)\right]\right\},
\end{aligned}
$$

where $\varrho_{\mathrm{ph}}$ is the photospheric mass density and $s_{\mathrm{ph}}$ enables us to control the smoothness of the transition region. We set $\varrho_{\mathrm{ph}}=$ $10^{5} \varrho_{\mathrm{e} 0}$ and $s_{\mathrm{ph}}=2 \mathrm{Mm}$. A similar expression is implemented for the curved slab, namely

$\varrho_{0}(z)=\varrho_{\mathrm{e}}(z)+\frac{1}{2}\left(\varrho_{\mathrm{ph}}-\varrho_{\mathrm{e}}(z)\right)\left[1-\tanh \left(\frac{z}{s_{\mathrm{ph}}}\right)\right]$.

Initial mass density profiles for these slabs are displayed in Fig. 3.

\section{Numerical results for slabs oscillations}

In this section we present results of numerical simulations of the straight and curved slabs described in the former part of this paper. We adopt the numerical code Athena developed by Gardiner \& Stone (2005). This code implements the higherorder Godunov method for solving time-dependent ideal magnetohydrodynamic equations. The algorithm is based on the piecewise parabolic method and it is second-order accurate in space and time. Equation (13) is satisfied by using a constrained transport scheme.

Equations (9)-(13) are solved numerically in Eulerian boxes with the $x$ - and $z$-dimensions $(-0.2 L, 1.2 L) \times(-0.6 L, 0.6 L)$ for a case of the straight slab and $(-L, L) \times(-0.2 L, 1.6 L)$ for the curved slab. These boxes are covered by a uniform grid of $500 \times 500$ numerical cells. Grid convergence studies showed that this resolution is sufficient for deriving results independent of
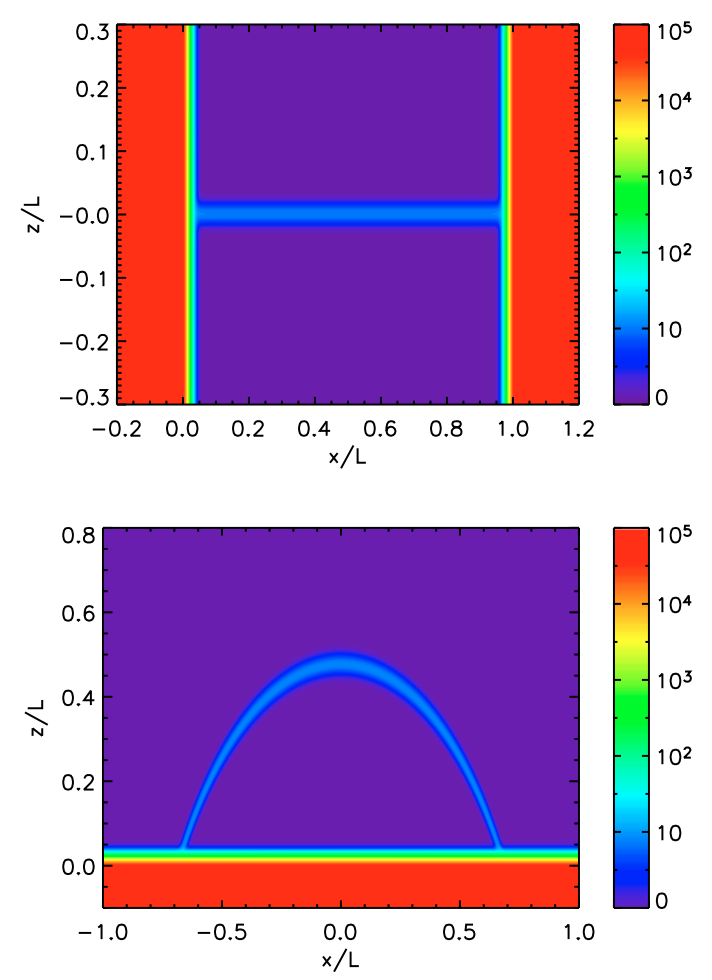

Fig. 3. Initial mass density profiles for the straight (top) and curved (bottom) slabs. The density (color bar, logarithmic scale) is given in arbitrary units $\left(\varrho_{\mathrm{e} 0}=10^{-12} \mathrm{~kg} \mathrm{~m}^{-3}\right)$.

the grid. We apply open boundary conditions for all perturbed plasma variables at all boundaries to allow a wave signal to leave freely the simulation region.

\subsection{A straight slab}

For the straight slab (Fig. 3, left panel), we launch an initial pulse in the vertical component of velocity:

$V_{z}(x, z, t=0)=A_{V} \mathrm{e}^{-\left[\left(x-x_{0}\right) / w_{x}\right]^{2}} \mathrm{e}^{-\left[\left(z-z_{0}\right) / w_{z}\right]^{2}}$.

Here $A_{V}=0.3 V_{\mathrm{A}}$ is the amplitude of the pulse of its width $w_{x}=0.35 \mathrm{~L}$ and $w_{z}=0.15 \mathrm{~L}$. We set $z_{0}=-0.05 \mathrm{~L}$ and test several positions of $x_{0}$ in determining the most suitable location for triggering the mode $n=2$. Some fast magnetoacoustic kink waves excited by the initial pulse of Eq. (21) are trapped between two photospheric layers located at the ends of the slab. Figure 4 displays time signatures of mass density evaluated at both $x=L / 4$ (left panel) at $x=L / 2$ (right panel) for $x_{0}=L / 4$ (Fig. 3). These time signatures should be compared with the time signatures of the oscillating string (Fig. 2, top panels). The time signatures of Fig. 4 exhibit oscillations that result essentially from fast magnetoacoustic kink waves. From the complexity of these signals, we infer that the fundamental mode is not the only wave excited in the system. A Fourier power spectra analysis of these time signatures confirm a presence of two different waveperiods of oscillations in the system (Fig. 5); a waveperiod of the fundamental mode $P_{1} \simeq 200 \mathrm{~s}$ and a waveperiod of the first harmonic mode $P_{2} \simeq 100 \mathrm{~s}$. These values agree with the analytical formula given by Eq. (8). By comparing Figs. 5 and 2 (bottom panels), we infer that we have triggered oscillations akin to the string oscillations of Eq. (1). It is noteworthy that more power is accumulated in the fundamental mode than in its first harmonic. 

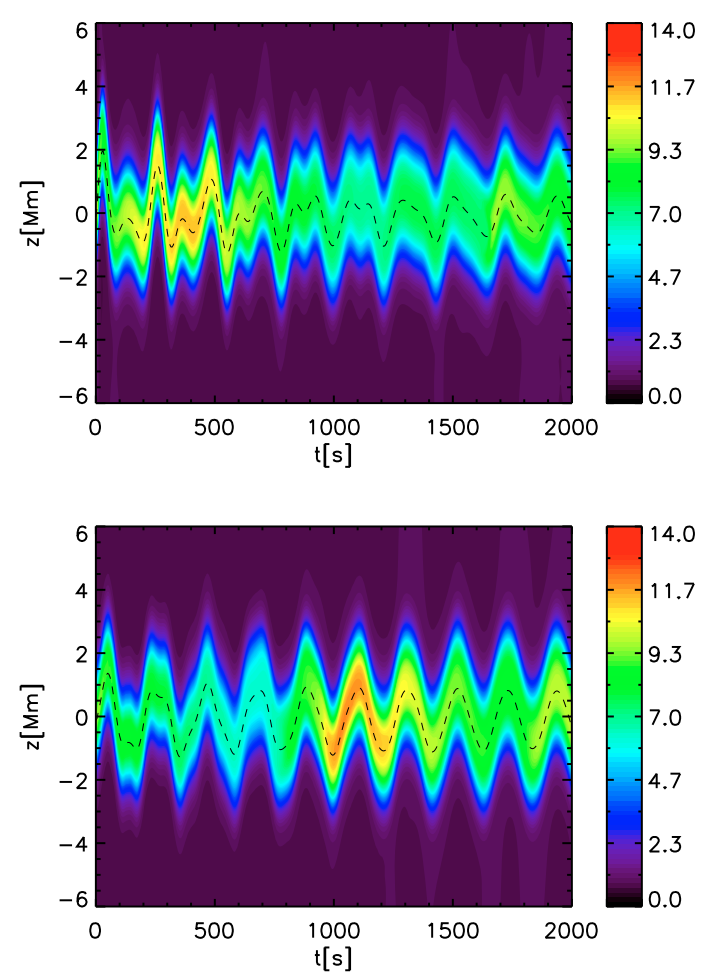

Fig. 4. Time signatures of mass density (color bar, units of $\varrho_{\mathrm{e}}$ ), excited by the velocity pulse of Eq. (21) at $x_{0}=L / 4, z_{0}=-0.15 \mathrm{~L}$. Top (bottom) panel corresponds to the detection region along the line $x=L / 4$ ( $x=$ $L / 2$ ). Dashed lines correspond to the positions of the slab center.

Figure 6 displays the positions of the estimated straight slab center at different moments of time. It is discernible that the horizontal position of the node varies in time, which agrees with the analytical findings of Fig. 1.

\subsection{A curved slab}

In the case of the curved slab, a more realistic structure than the straight slab discussed above, we launch a Gaussian pulse in the velocity

$\boldsymbol{V}(x, z, t=0)=A_{V}[-1,0,1] \mathrm{e}^{-\left[\left(x-x_{0}\right) / w_{x}\right]^{2}} \mathrm{e}^{-\left[\left(z-z_{0}\right) / w_{z}\right]^{2}}$.

Here $A_{V}=0.4 V_{\mathrm{A}}(z=0)$ is the amplitude of the pulse that has width $w_{x}=0.35 \mathrm{~L}$ and $w_{z}=0.15 \mathrm{~L}$. We launch the pulse along the line $z_{0}=-x_{0}$ and trace mass density profiles along the line $x=0$ close to the slab summit, where the node of the first harmonic mode $(n=2)$ of vertical kink oscillations is present, and along the line $x=-0.4 L$, where the antinode of this mode is located approximately. With the information gathered from these detecting lines, we can determine which mode of vertical kink oscillations is generated.

Typical time signatures that result from the velocity pulse are shown in Fig. 7. After a significant initial shift (top panel), the curved slab begins oscillating. Mass density profiles exhibit kink oscillations for which the node changes its position close to the slab apex (Fig. 8). This behavior agrees with the analytical solution for both string oscillations (Fig. 1) and a straight slab (Fig. 6). The other two nodes are located at the footpoints.

Fourier power spectra are displayed in Fig. 9. In a detection region along the line $x=-0.4 L$ (top panel), two waveperiods $P_{1}=360 \mathrm{~s}$ and $P_{2}=190 \mathrm{~s}$ are discovered. These waveperiods
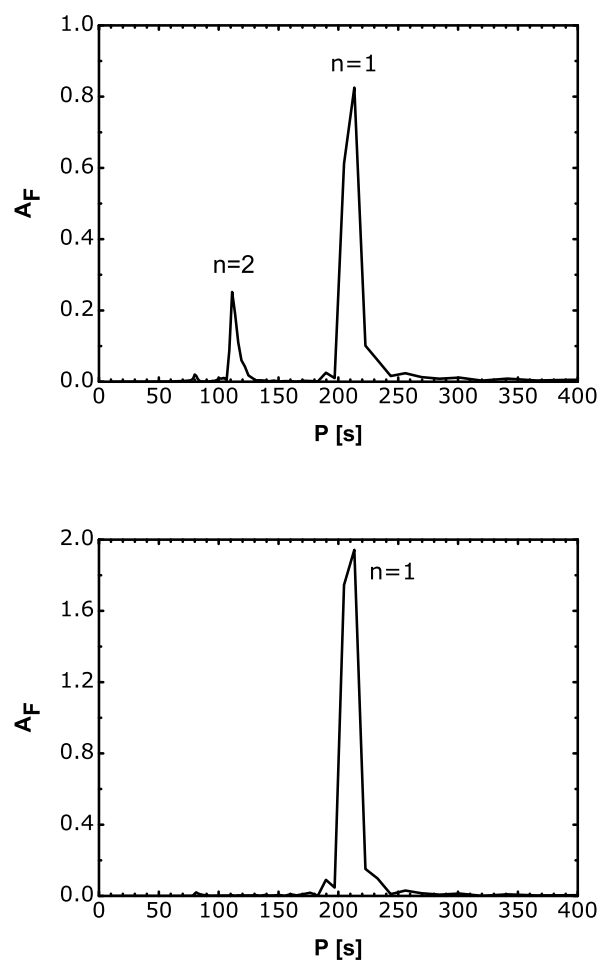

Fig. 5. Fourier power spectra of the wave signals of Fig. 4.

correspond to the modes of $n=2$ and $n=1$, where more power is associated with the fundamental mode. The time signature for the detection region located at the apex of a slab corresponds to the fundamental mode $(n=1)$ only. The difference in waveperiods between the straight and curved slabs is caused by the different values of the slab lengths and Alfvén speeds.

\section{Summary}

We have presented numerical results for impulsively generated oscillations of a coronal loop modeled by either a straight slab or an arcade slab.

It was shown by Gruszecki \& Murawski (2008) that the effect of gravity produces a decrease in the wave period and an increase in the attenuation time. This decrease is a consequence of a higher Alfvén speed within the gravitationally-stratified arcade loop, while the weaker attenuation reflects a smaller amount of wave tunneling than in the gravity-free case.

Our findings apply to the gravity-free case and can be summarized as follows. The initial pulse of momentum initiated offcenter at the beginning of the simulation triggers vertical oscillations consisting of the fundamental and first-harmonic modes. A node of these oscillations is not fixed in time but it moves about the loop apex. These numerically obtained results agree with the analytical findings for simple models of a coronal loop, such as an elastic string and a straight slab.

The simultaneous presence of the fundamental and first harmonic modes in a coronal loop explains the difficulty in detecting the latter mode; as a result of a lower power accumulated in the first harmonic mode, the latter is more difficult to detected in the coronal loop. Reports of these oscillations are indeed rare (Verwichte et al. 2004; de Moortel \& Brady 2007; van Doorsselaere et al. 2007). 

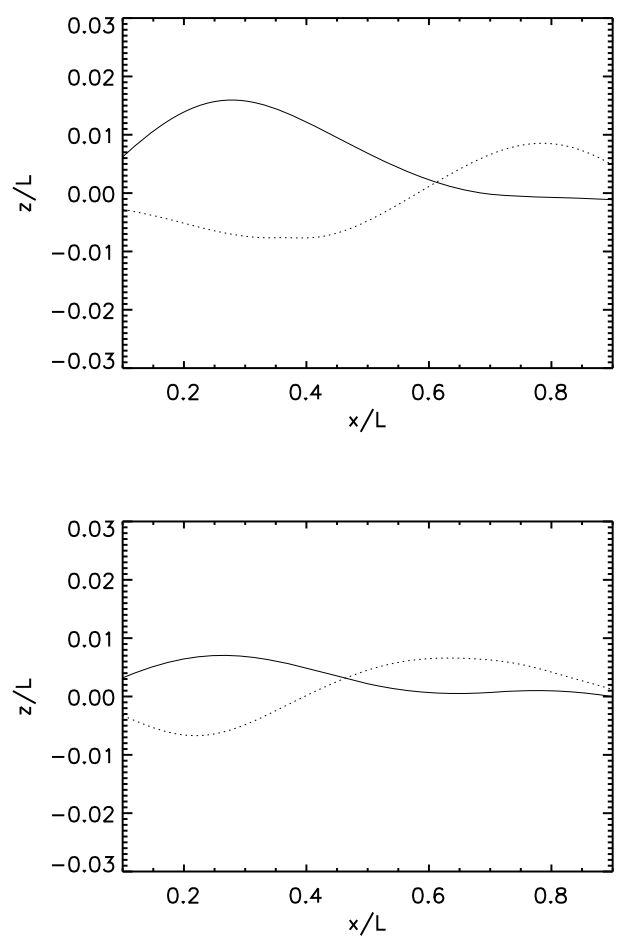

Fig. 6. Positions of the straight slab center for a case of Fig. 4. Top panel: solid (dotted) line corresponds to $t=100 \mathrm{~s}(t=260 \mathrm{~s})$. Bottom panel: $t=305 \mathrm{~s}$ (solid line) and $t=510 \mathrm{~s}$ (dotted line).
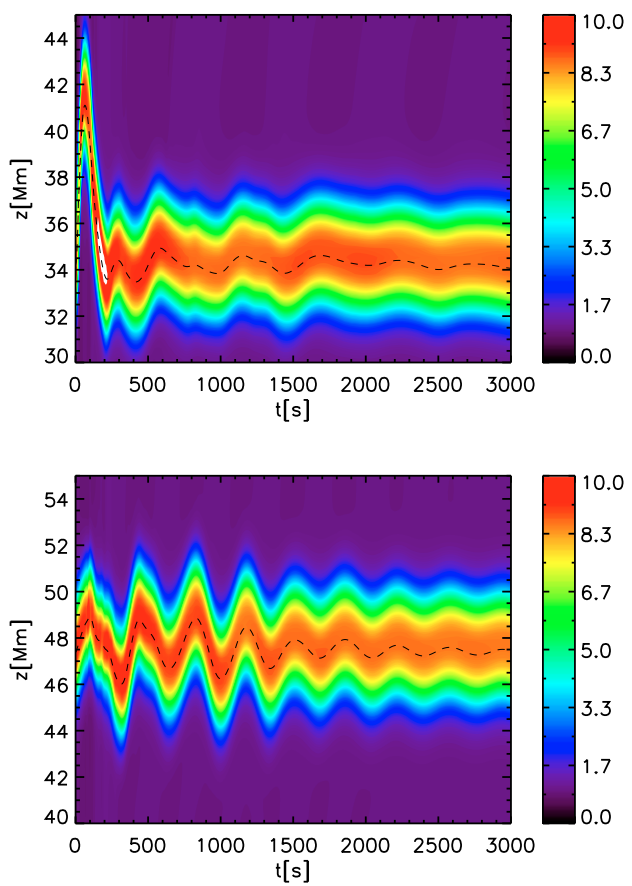

Fig. 7. Time signatures of mass density (color bar, units of $\varrho_{\mathrm{e} 0}$ ) for $z_{0}=$ $-x_{0}=L / 2$ (Eq. (22)). These time signatures are collected along the line $x=-0.4 L$ (top panel) and $x=0$ (bottom panel).

Acknowledgements. KM's work was supported by the grant for years 20072010 from the Polish Ministry of Science. The magnetohydrodynamics code used in this study was developed at Princeton University by Tom Gardiner, Jim Stone, Peter Teuben and John Hawley with support of the NSF Information
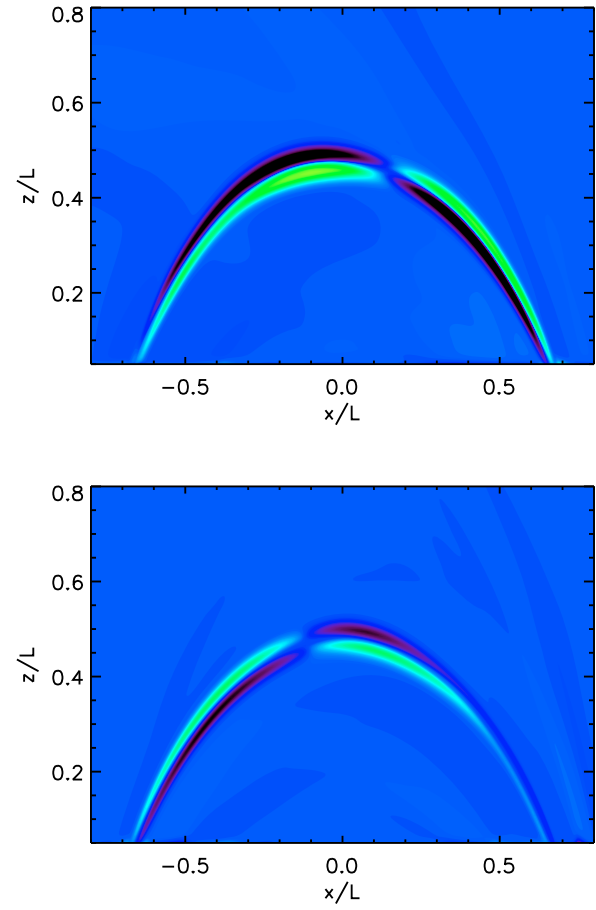

Fig. 8. Mass density profiles for the case of curved slab oscillations shown in Fig. 7. Top panel: dark (bright) pattern corresponds to the position of the loop at $t=140 \mathrm{~s}(t=300 \mathrm{~s})$. Bottom panel: same profiles at $t=410 \mathrm{~s}$ and $t=580 \mathrm{~s}$.
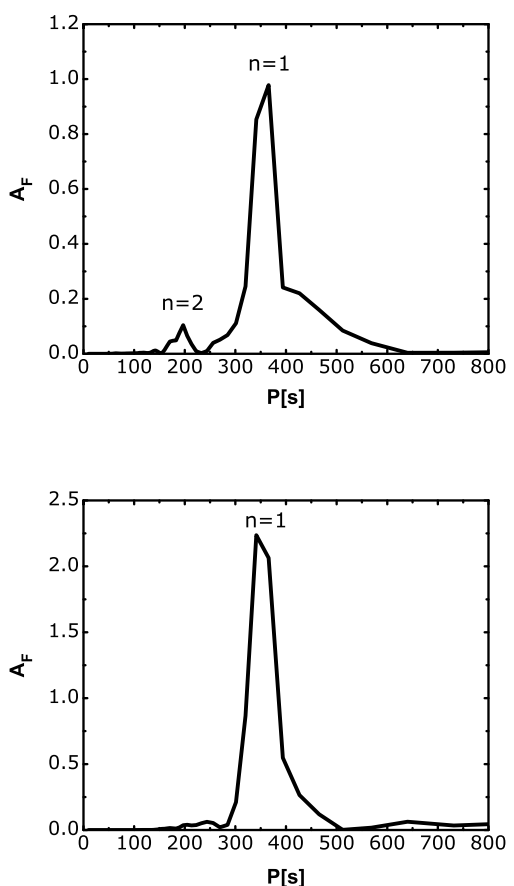

Fig. 9. Fourier power spectra of the wave signals shown in Fig. 7. Top panel corresponds to the detection region at $x=-0.4 \mathrm{~L}$ while bottom panel to $x=0$.

Technology Research (ITR) program. KM's work was supported by the grant from the Polish Ministry of Science for years 2007-2010. 


\section{References}

Aschwanden, M., Fletcher, L., Schrijver, C., \& Alexander, D. 1999, ApJ, 520, 880

Aschwanden, M., Nakariakov, V., \& Melnikov, V. F. 2004, ApJ, 600, 458

Brady, C. S., \& Arber, T. D. 2005, A\&A, 438, 733

De Moortel, I., \& Brady, C. S. 2007, ApJ, 664, 1210

De Moortel, I., Ireland, J., Walsh, R. W., \& Hood, A. W. 2002, Sol. Phys., 209, 61

Díaz, A. J., Zaqarashvili, T., \& Roberts, B. 2006, A\&A, 455, 709

Gardiner, T. A., \& Stone, J. M. 2005, J. Comput. Phys., 205, 509

Gruszecki, M., \& Murawski, K. 2008, A\&A, 487, 717

Gruszecki, M., Murawski, K., \& McLaughlin, J. 2008, A\&A, 489, 413
Nakariakov, V. M., Ofman, L., Deluca, E. E., Roberts, B., \& Davila, J. M. 1999, Science, 285, 862

Nakariakov, V. M., Melnikov, V. F., \& Reznikova, V. E. 2003, A\&A, 412, 7 Ofman, L. 2007, ApJ, 655, 1134

Ofman, L., \& Wang, T. J. 2008, A\&A, 482, L9

Roberts, B., Edwin, P. M., \& Benz, A. O. 1984, ApJ, 279, 857

Schrijver, C. J., Aschwanden, M. J., \& Title, A. M. 2002, Sol. Phys., 206, 69

Selwa M., Murawski, K., Solanki, S. K., \& Wang, T. J. 2007, A\&A, 462, 1127

van Doorsselaere, T., Nakariakov, V. M., \& Verwichte, E. 2007, A\&A, 473, 959

Verwichte, E., Nakariakov, V. M., Ofman, L., \& DeLuca, E. E. 2004, Sol. Phys., 223,77

Wang, T. J., \& Solanki, S. K. 2004, A\&A, 421, L33

Wang, T. J., \& Solanki, S. K., Innes, D. E., \& Curdt, W. 2005, A\&A, 435, 753 